\title{
Coverage of the requirements of first and second level stroke unit in Italy
}

\author{
Monica Bandettini di Poggio ${ }^{1}$ (1) Danilo Toni ${ }^{2} \cdot$ Carlo Gandolfo $^{1} \cdot$ Damiano Paolicelli $^{3} \cdot$ Andrea Zini $^{4} \cdot$ Elio Agostoni $^{5}$. \\ Fabio Bandini ${ }^{6}$. Michele Ragno ${ }^{7}$. Maria Concetta Altavista ${ }^{8}$. Antonio Bertolotto ${ }^{9}$. Gabriele Siciliano ${ }^{10}$. \\ Michele Vecchio ${ }^{11}$. Nicola Tambasco ${ }^{12}$. Antonio Gambardella ${ }^{13}$. Paolo Manganotti ${ }^{14}$. Maurizio Melis ${ }^{15}$. \\ Marco Onofrj ${ }^{16}$. Giuseppe De Michele ${ }^{17} \cdot$ Nicoletta Reale $^{18} \cdot$ Gioacchino Tedeschi $^{19} \cdot$ GianLuigi Mancardi $^{20}$
}

Received: 1 June 2020 / Accepted: 19 July 2020 / Published online: 31 July 2020

(C) The Author(s) 2020

\begin{abstract}
Background and aim In the scientific literature, there is unanimous consensus that hospitalization in stroke unit (SU) is the most important treatment for stroke patients. In this regard, the Act number 70/2015 by the Italian government identified specific skills that contribute to a classification of SU and outlined a "hub and spoke" stroke network. The aim of our study was to check the coverage of requirements of first and second level SU in the national territory and to shed light on any deficit or misdistribution of resources.

Material and methods In 2019, a survey on the current situation related to stroke care in Italy was carried out by the Italian Society of Neurology (SIN), The Italian Stroke Organization (ISO), and the Association for the Fight against Stroke (A.L.I.Ce). Results First level SU was found to be 58 against a requirement, according to the Act 70/2015, of 240. Second level SU was found to be 52 compared with an expected requirement of 60 . Neurointerventionists were 280 nationally, with a requirement of 240. A misdistribution of resources within individual regions was often seen.

Conclusions The survey demonstrated a severe shortage of beds dedicated to cerebrovascular diseases, mainly because of lack of first level SU, especially in central and southern Italy. It also suggests that the current hub and spoke system is not yet fully implemented across the country and that resources should be better distributed in order to ensure uniform and fair care for all stroke patients on the whole territory.
\end{abstract}

Keywords Stroke $\cdot$ Stroke unit $\cdot$ Neurointerventionists $\cdot$ Thrombolysis

\section{Introduction}

In the scientific literature, there is unanimous consensus that hospitalization in stroke unit (SU) is the most important treatment for the generality of stroke patients. The benefit in terms of better long-term outcome of acute stroke patients admitted into $\mathrm{SU}$ versus conventional wards has been previously demonstrated in several randomized trials and their meta-analysis [1]. Two Italian observational follow-

Electronic supplementary material The online version of this article (https://doi.org/10.1007/s10072-020-04616-x) contains supplementary material, which is available to authorized users.

Monica Bandettini di Poggio

monicabandettini@gmail.com

Extended author information available on the last page of the article up studies confirmed SU patient management as predictor of good outcome in a real-world setting, across all age ranges and clinical characteristics [2,3]. In this regard, both European and Italian guidelines suggest transporting all cases of suspected stroke to the emergency room of the nearest hospital provided with SU $[4,5]$. The Act number $70 / 2015$ by the Italian government identified specific skills that contribute to a classification of stroke unit (Table 1). First level SU are characterized by the presence of at least one dedicated neurologist, a committed nursing staff, at least one bed with continuous monitoring, and the possibility of carrying out intravenous thrombolytic therapy (IVT) and connection with a 2 nd level stroke center. Second level SU must treat at least 500 stroke cases/year, have dedicated staff $24 \mathrm{~h}$ a day, have neuroradiology active 24/7, and be able to perform mechanical thrombectomy in emergency (Table 1). The evidence is that stroke care involves 
Table 1 Classification of stroke unit as defined by the current Act $70 / 2015$ of the Italian health government

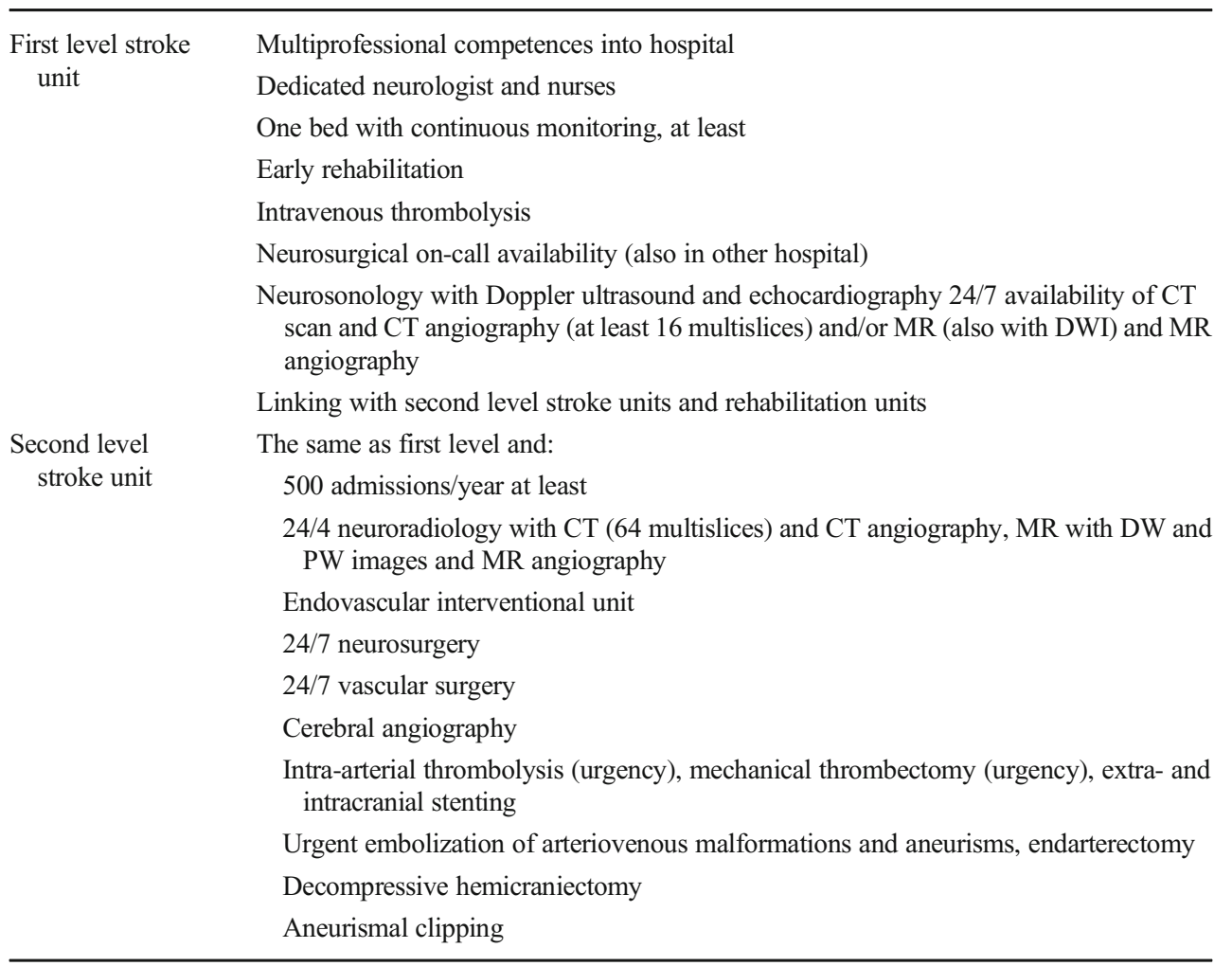

management by a dedicated stroke team consisting of vascular neurologist, neurosurgeon, neurointerventionist, radiologist, anesthesiologist, specialized nurses, other trained medical personnel, and rehabilitation facilities. It also requires the availability of cutting-edge technology at their disposal. Since this requires high costs and it is impossible to have all these facilities in every hospital in a given area, the government Act 70/2015 identified a "hub and spoke" stroke network. According to this model, a single hospital staffed by specialized physicians and with the appropriate high-technology infrastructure forms the hub (second level emergency departments-EDs), while other hospitals providing less complex forms of care act as the spoke (first level EDs). The Act number 70/2015 states also that a first level SU must be located in a first level ED, with a catchment area of between 150,000 and 300,000 inhabitants, and a second level SU must be located in the second level EDs, with a catchment area of between 600,000 and 1,200,000 inhabitants. Made these premises, the need in Italy for first level stroke centers is 240 and for second level stroke centers is 60 , which should be adequately and rationally distributed throughout the national territory. Referring to the "Notebooks of the Ministry of Health on the Organization of Stroke Assistance: The Stroke Units" n.2 of MarchApril 2010, the need for dedicated beds is about 8 beds in SU every 150-300,000 inhabitants, corresponding to about 1 bed every 19,000 inhabitants.

\section{Aim}

The aim of this study was to check the coverage of the requirements of first and second level SU in the national territory and to shed light on any deficit or misdistribution of resources.

\section{Material and methods}

In 2019, the Italian Society of Neurology (SIN), in collaboration with A.L.I.Ce. (Association for the Fight against Stroke) and ISO (The Italian Stroke Organization), carried out a study on the current situation related to stroke care in Italy, region by region. Detailed information was collected by regional secretaries of SIN.

The estimate of possible deficiencies was based on the terms of the Act 70/2015, even if for this census, we used a more permissive method, knowing in advance that the healthcare situation in our country was not generally totally overlapping with ministerial indications [6]. We therefore decided that minimum requirements to meet the criterion for first level SU should be (a) the presence of at least one dedicated neurologist or (not "and") specialized nurses; (b) at least one bed with continuous monitoring; and (c) capability of performing intravenous thrombolysis (IVT) $24 / 7$. On the other hand, we considered second level SU all those centers able to perform mechanical thrombectomy (MT) 24/7 with a team of 
neurointerventionists of at least 4 units, even if other criteria set out in the Act 70/2015 were not completely met.

Using this method, we separately analyzed (a) the number of first level and second level SU; (b) the number of beds in SU (beSU); (c) the number of stroke beds in traditional wards such as EDs or neurological departments (beTW); (d) the total number of beds dedicated to stroke (TObe); and (e) the number of neurointerventionists able to perform mechanical thrombectomies.

To obtain this information, we created a CRF to be sent to all regional SIN secretaries who were asked to fill in the requested information fields, city by city. Data were then delivered to the administrative office of SIN and were carefully reviewed by authors of this study.

\section{Results}

First level SU (as defined by our "permissive" method) was found to be 58 against a requirement, according to the Act 70/ 2015, of 240. Second level SU (as defined by our "permissive" method) was found to be 52 compared with an expected requirement of 60 .

The number of beds available in SU (beSU) was 723, compared with an expected of 1920 . When we also considered those beds dedicated to acute cerebrovascular disorders in traditional wards (beTW) outside SU, the total number of beds increased to 1176 (beSU + beTW), always lower than the need as established by the Ministry of Health (1920 beds).

Neurointerventionists (NIs) (in the vast majority neuroradiologists or radiologists) were 280 nationally, with a requirement of 240 .

Detailed data, region by region, are available as supplementary material.

In Figs. 1 and 2, we have depicted each region with a different color depending on the degree of shortage of beds dedicated to stroke and of NIs compared with the expected. Red color indicates that the shortage is $51 \%$ greater than the requirement, orange between 50 and $31 \%$, yellow between 30 and $11 \%$, and green $\leq 10 \%$. The results indicate a severe shortage of dedicated beds to cerebrovascular patients in central and southern Italy. In addition, we have often seen a misdistribution of resources within individual regions. As it is shown in the supplementary material, significant differences in coverage are not uncommon depending on the provinces. This applies to stroke unit beds and even more to neurointerventionist services. So, regions that appear to be "green" in the map still present problems within them.

\section{Discussion}

This survey shows a severe shortage of beds dedicated to cerebrovascular diseases, mainly because of lack of first level SU. In front of a requirement of about 240 first level SU, our census found only 58 of them, resulting in a shortfall of $76 \%$. More generally when we refer to beds dedicated to cerebrovascular patients, the deficiency is still about $40 \%$. The lack of SU is particularly disadvantageous because of its known benefit in terms of better assistance and long-term outcome.

In fact, if the treatment of choice for ischemic stroke patients is IVT in combination eventually with MT, the shortage of first and second level SU might explain, at least in part, the rate of patients who undergo reperfusion therapies in our country. As proof of this, we compared the rate of IVT in a certain region (data extracted from the Safe Implementation of Treatments in Stroke (SITS) Registry) with the rate of available SU in the same area and noticed a certain correspondence (www.sitsinternational.org). For example, for Liguria and Tuscany, which resulted in our study with a shortage of cerebrovascular beds $\leq 10 \%$ than the required, data from the SITS showed a high rate of IVT. By contrast, data from the SITS are less encouraging for regions such as Lazio and Campania, depicted in red on our map in Fig. 1.

However, the beneficial effects of SU admission are not depending only on the higher probability of being treated with reperfusional therapies, but mainly on the patient management in SU independently [2]. As it is known, the benefit comes from the presence of multidisciplinary team with expert vascular neurologists and dedicated nurses, better parameter monitoring in the acute phase and early access to intensive rehabilitation. Unfortunately, in Italy, evidence of SU deficiency has been known for at least 15 years. For example, in 2006, data from a survey carried out by the PROSIT study group showed that in $2003 / 04$, only $9 \%$ of the hospital services had organized SU care [6]. Since then, the number of SUs increased thanks to the approval of intravenous thrombolysis only in that setting, and the quality of acute stroke care improved, but the situation has changed only partially and inhomogenously among different regions. In this scenario, we believe that a major health policy maneuver supported by national legislation is required in order to adjust national stroke care to what is required by the ministerial Act 70/2015. In fact, in the era of thrombectomy, it is important that we keep a population perspective in order to appropriately treat most stroke patients who only require medical treatment. In fact, although every patient with stroke is eligible for dedicated stroke unit care, some $25 \%$ of the patients will be eligible for IVT, and only an estimated $10 \%$ will be eligible for MT [7].

In this desirable healthcare reform, efforts should be made also to increase the availability of neurologist dedicated to stroke (vascular neurologist, VN) in order to break the vicious circle of general neurologists being uncomfortable with managing acute stroke patients because of lack of enough exposure and experience. A VN should be a specialist in neurology 


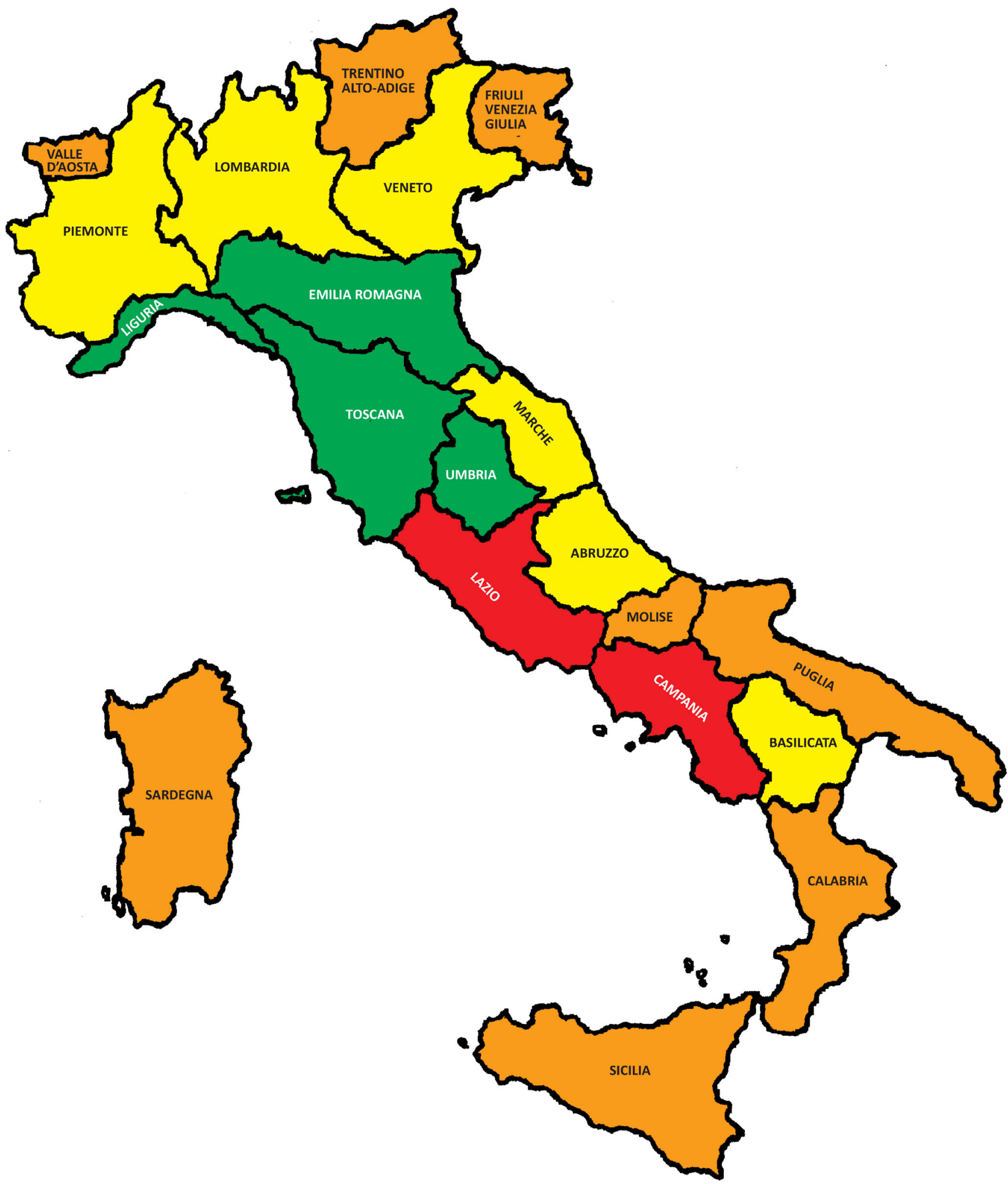

Fig. 1 Extent of the lack of beds dedicated to stroke compared with the expected in Italy

with a profound knowledge on stroke causes, its pathogenic mechanisms, and therapeutic possibilities. He should have extensive clinical skills for facing the complex profile of acute stroke patients and not only the manual ability to recanalize the occluded arterial vessel. Even if our study does not have the power to estimate the lack of VNs, recent data have been published on this topic [8], and in agreement with the authors, we believe that progress in the management of acute stroke 


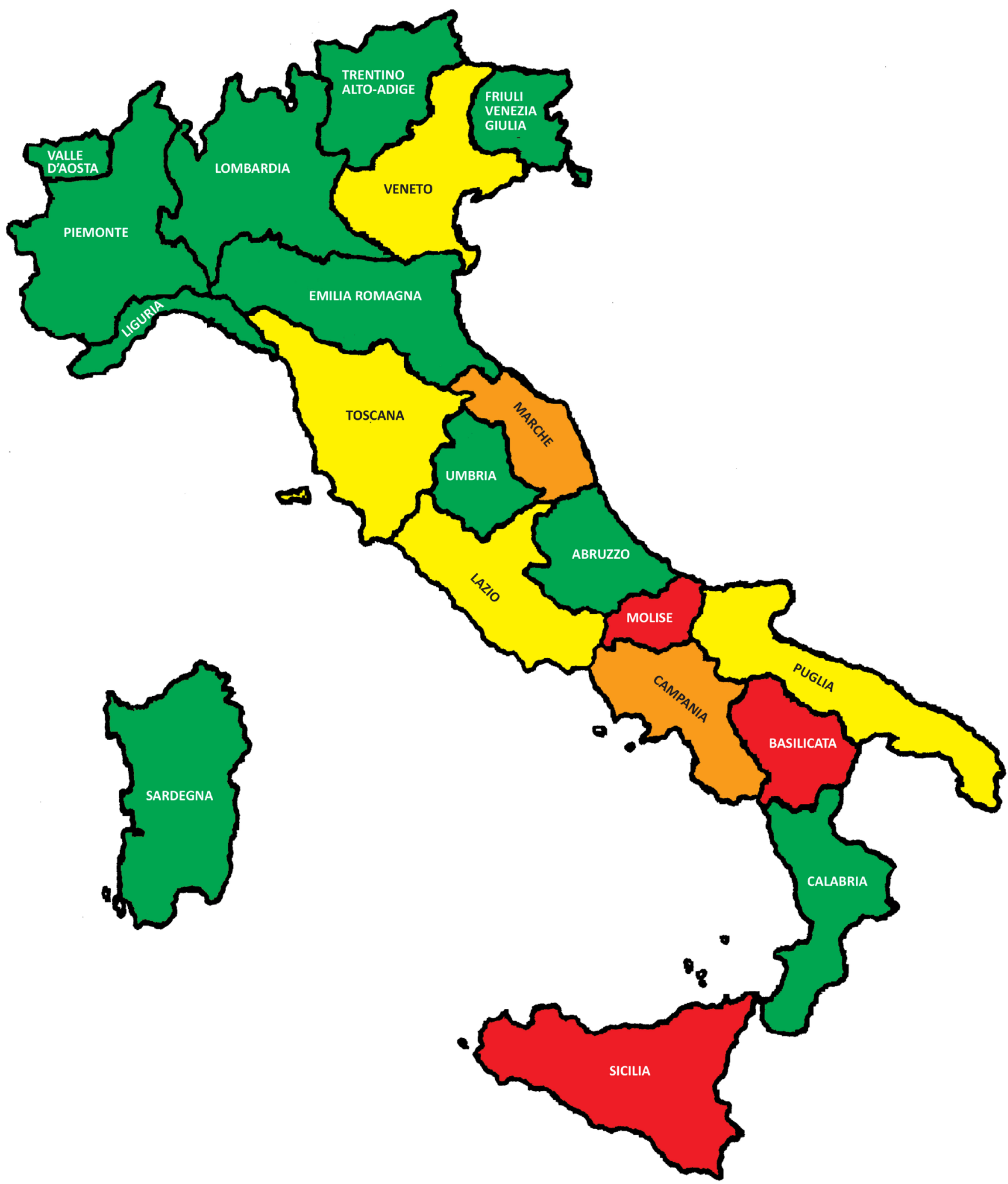

Fig. 2 Extent of the lack of neurointerventionists in Italy

has the risk of becoming meaningless if there is no workforce able to implement those standards.

On the other hand, our survey found that the number of NIs was higher than expected, theoretically suggesting that endovascular recanalization should be appropriately managed in our country. Unfortunately, the high number of NIs is not synonymous with optimal 24/7 coverage of NIs services. Main potential causes of this partial ineffectiveness of the 
system are (a) the presence of a number of NI services higher than required in some regions, but with sufficient staff only in some SU (considering a minimum of at least 4 NIs per center to provide the necessary coverage 24/7); (b) misdistribution of services in the regional territory with disparities in coverage depending on the provinces (e.g., in Lazio; see supplementary material); and (c) absence or extreme deficiency of endovascular services in some regions (e.g., in Molise, Basilicata, Sicilia).

This survey suggests that the current hub and spoke system is not yet fully implemented across the country and that NI resources should be better distributed in order to ensure uniform and fair care for all stroke patients on the whole territory. Our study was not designed with the aim to identify the discipline of origin of NIs (radiology, neuroradiology, or neurology) and their average age. Even if our available data show that interventionists mostly adhere to neuroradiology, a better characterization of national NIs should be object of further surveys. Considering the extremely fast growth of interventional neuroradiology and the inevitable generational change that will take place in the next years, a European neuroradiological multi-society group recently set up recommendations for the development of MT services, including consensus on the minimum requirements for centers providing such treatment and training requirements in interventional neuroradiology [9]. Despite differences in resources across Italian healthcare facilities, we believe that efforts should be made to implement these recommendations as a standard of care.

Acknowledgments We thank the organizational secretariat of SIN, Siena Congress, and in particular Dr. Barbara Frati for the help in data collection.

\section{Compliance with ethical standards}

Conflicts of interest The authors declare that they have no conflict of interest.

Ethical approval This is a retrospective study focused on health organization issues. For this purpose we did not use any clinical or biological data of patients and therefore formal approval from ethic commitee was not requested

Open Access This article is licensed under a Creative Commons Attribution 4.0 International License, which permits use, sharing, adaptation, distribution and reproduction in any medium or format, as long as you give appropriate credit to the original author(s) and the source, provide a link to the Creative Commons licence, and indicate if changes were made. The images or other third party material in this article are included in the article's Creative Commons licence, unless indicated otherwise in a credit line to the material. If material is not included in the article's Creative Commons licence and your intended use is not permitted by statutory regulation or exceeds the permitted use, you will need to obtain permission directly from the copyright holder. To view a copy of this licence, visit http://creativecommons.org/licenses/by/4.0/.

\section{References}

1. Stroke Unit Trialists' Collaboration (2013) Organised inpatient (stroke unit) care for stroke. Cochrane Database Syst Rev 9: CD000197. https://doi.org/10.1002/14651858.CD000197.pub3

2. Candelise L, Gattinoni M, Bersano A, Micieli G, Sterzi R, Morabito A, on the behalf of the PROSIT Study Group (2007) Stroke-unit care for acute stroke patients: an observational follow-up study. Lancet 369:299-305

3. Di Carlo A, Lamassab M, Wellwood I, Bovis F, Baldereschi M, Nencini P, Poggesi A, Cramaro A, Pescini F, Lucente G, Wolf CDA, Inzitari D, for the European Registers of Stroke (EROS) Project* (2011) Stroke unit care in clinical practice: an observational study in the Florence center of the European registers of stroke (EROS) project. Eur J Neurol 18:686-694

4. Ahmed N, Audebert H, Turc G, Cordonnier C, Christensen H, Sacco S, Sandset EC, Ntaios G, Charidimou A, Toni D, Pristipino C, Köhrmann M, Kuramatsu JB, Thomalla G, Mikulik R, Ford GA, Martí-Fàbregas J, Fischer U, Thoren M, Lundström E, Rinkel GJ, van der Worp HB, Matusevicius M, Tsivgoulis G, Milionis H, Rubiera M, Hart R, Moreira T, Lantz M, Sjöstrand C, Andersen G, Schellinger P, Kostulas K, Sunnerhagen KS, Keselman B, Korompoki E, Purrucker J, Khatri P, Whiteley W, Berge E, Mazya M, Dippel DW, Mustanoja S, Rasmussen M, Söderqvist ÅK, Escudero-Martínez I, Steiner T (2019) Consensus statements and recommendations from the ESO-Karolinska Stroke Update Conference, Stockholm 11-13 November 2018. Eur Stroke J. 4(4):307-317

5. Italian Stroke Organisation (ISO)-SPREAD guidelines study group, ISO SPREAD (2017) Linee Guida Italiane per la diagnosi, prevenzione e trattamento dell'ictus cerebrale. Ed VIII

6. Bersano A, Candelise L, Sterzi R, Micieli G, Gattinoni M, Morabito A (2006) And the PROSIT study group stroke unit care in Italy. Results from PROSIT (project on stroke Services in Italy). A nationwide study. Neurol Sci 27:332-339

7. Leira EC, Savitz SI (2018) In the era of thrombectomy, let us also protect the majority of patients with stroke who only require medical treatment! Stroke. 49:1538-1540

8. Leira EC, Kaskie B, Froehler MT, Adams HP Jr (2013) The growing shortage of vascular neurologists in the era of health reform planning is brain! Stroke. 44:822-827

9. Pierot L, Jayaraman MV, Szikora I, Hirsch JA, Baxter B, Miyachi S, Mahadevan J, Chong W, Mitchell PJ, Coulthard A, Rowley HA, Sanelli PC, Tampieri D, Brouwer PA, Fiehler J, Kocer N, Vilela P, Rovira A, Fischer U, Caso V, van der Worp B, Sakai N, Matsumaru Y, Yoshimura SI, Anxionnat R, Desal H, Biscoito L, Pumar JM, Diaz O, Fraser JF, Linfante I, Liebeskind DS, Nogueira RG, Hacke W, Brainin M, Yan B, Soderman M, Taylor A, Pongpech S, Tanaka M, Terbrugge K, Asian-Australian Federation of Interventional and Therapeutic Neuroradiology (AAFITN); Australian and New Zealand Society of Neuroradiology (ANZSNR); American Society of Neuroradiology (ASNR); Canadian Society of Neuroradiology (CSNR); European Society of Minimally Invasive Neurological Therapy (ESMINT); European Society of Neuroradiology (ESNR); European Stroke Organization (ESO); Japanese Society for NeuroEndovascular Therapy (JSNET); French Society of Neuroradiology (SFNR); Ibero-Latin American Society of Diagnostic and Therapeutic Neuroradiology (SILAN); Society of NeuroInterventional Surgery (SNIS); Society of Vascular and Interventional Neurology (SVIN); World Stroke Organization (WSO) (2018) Standards of practice in acute ischemic stroke intervention: International Recommendations. AJNR Am J Neuroradiol. 39(11):E112-E117

Publisher's note Springer Nature remains neutral with regard to jurisdictional claims in published maps and institutional affiliations. 


\section{Affiliations}

Monica Bandettini di Poggio ${ }^{1}$ (D) Danilo Toni ${ }^{2} \cdot$ Carlo Gandolfo $^{1} \cdot$ Damiano Paolicelli $^{3} \cdot$ Andrea Zini $^{4} \cdot$ Elio Agostoni $^{5}$. Fabio Bandini ${ }^{6}$. Michele Ragno ${ }^{7}$. Maria Concetta Altavista ${ }^{8}$ - Antonio Bertolotto ${ }^{9} \cdot$ Gabriele Siciliano $^{10}$. Michele Vecchio ${ }^{11}$ - Nicola Tambasco ${ }^{12}$ - Antonio Gambardella ${ }^{13}$. Paolo Manganotti ${ }^{14}$ - Maurizio Melis ${ }^{15}$. Marco Onofrj ${ }^{16}$. Giuseppe De Michele ${ }^{17}$ • Nicoletta Reale ${ }^{18} \cdot$ Gioacchino Tedeschi $^{19} \cdot$ GianLuigi Mancardi $^{20}$

1 Department of Neuroscience, Rehabilitation, Ophthalmology, Genetics, Maternal and Child Health (DINOGMI), Policlinic San Martino Hospital, University of Genova, Genoa, Italy

2 Neurovascular Unit, Policlinico Umberto I, Department of Neurology and Psychiatry, University of Rome, 'La Sapienza', Rome, Italy

3 Dipartimento di Scienze Mediche di Base, Neuroscienze ed Organi di Senso, Università degli Studi di Bari “A. Moro”, Bari, Italy

4 IRCCS Istituto di Scienze Neurologiche di Bologna, Maggiore Hospital, Bologna, Italy

5 Department of Neurology, Niguarda Ca' Granda Hospital, Milan, Italy

6 Department of Neurology, S.Paolo Hospital, Savona, Italy

7 Neurologia AV5, Ascoli Piceno-, San Benedetto del Tronto, Italy

8 UOC di Neurologia, ASL Roma 1, Presidio Ospedaliero San Filippo Neri, Rome, Italy

9 SCDO Neurologia \& CRESM, AOU San Luigi, Orbassano, Italy

10 Dipartimento di Medicina Clinica e Sperimentale, Clinica Neurologica, Università di Pisa e Azienda Ospedaliero Universitaria Pisana, Pisa, Italy

11 U.O. of Physical Medicine and Rehabilitation, Policlinico Vittorio Emanuele University Hospital, University of Catania, Catania, Italy
12 Clinica Neurologica, Azienda Ospedaliera e Universitaria di Perugia, Perugia, Italy

13 Medical and Surgical Sciences Department, School of Medicine, Magna Græcia University of Catanzaro, Viale Europa, Catanzaro, Italy

14 Clinical Unit of Neurology, Department of Medicine, Surgery and Health Sciences, Cattinara University Hospital ASUGI, University of Trieste, Trieste, Italy

15 SC Neurologia e Stroke Unit, Azienda Ospedaliera G.Brotzu, Cagliari, Italy

16 Clinica Neurologica, Dipartimento di Neuroscienze, Imaging e Scienze Cliniche, Università Chieti-Pescara, Chieti, Italy

17 Dipartimento di Neuroscienze e Scienze Riproduttive ed Odontostomatologiche, Università di Napoli Federico II, Naples, Italy

18 Association for the Fight against Stroke (A.L.I.Ce.) Italia Odv, Rome, Italy

19 Department of Advanced Medical and Surgical Sciences, University of Campania "Luigi Vanvitelli", Naples, Italy

20 Department of Neuroscience, Rehabilitation, Ophthalmology, Genetics, Maternal and Child Health, University of Genova and IRCCS ICS Maugeri, Genova, Pavia, Italy 